\title{
THE GALACTIC DISTRIBUTION OF O-RICH AGB STARS
}

\author{
B.W. JIANG and J.Y. HU \\ Beijing Observatory, Academia Sinica, Beijing, 100080, China
}

In 1976 Georgelin et al.(1976) combined optical and radio observational data of HII regions to study the structure of our Galaxy. The distance of each HII region can be obtained from its radio continuum flux caused by free-free emission and the flux of the Balmer lines with high accuracy. The results clearly show three arms and are used to draw the spiral structure of our Galaxy. It is commonly accepted now.

Habing(1988) pointed out that about $4 / 5$ of all AGB stars are located in the galactic disk. There are some advantage using AGB stars as a tool of studying the galactic structure: 1 . They are powerful infrared sources and mainly radiated in the infrared region. 2 . The interstellar extinction in IRAS wavelengths can be neglected. and 3. IRAS completed a whole sky survey, and it is possible to select a complete sample from IRAS PSC.

A sample of AGB stars with O-rich circumstellar envelopes is selected from the IRAS PSC in the regions of IIIa, IIIb and IV defined by van der Veen and Habing(1988). The distance for each object is obtained assuming that the luminosities of O-rich AGB stars are identical. The spatial distribution of the sample shows a similar spiral structure presented by Georgelin et al.(1976). This confirms that the O-rich AGB stars are a disk population and that they are predominantly located in the arms. Fitting with the spiral structure of Georgeling et al. we derive that the average luminosity of the objects in the sample is $8300 \mathrm{Lo}$. It is larger than $4000 \mathrm{Lo}$ derived by Habing(1988). We consider this is result of selective effect. According to the dynamics of the Galaxy, only AGB stars with $\mathrm{M}_{0}$ larger than 2-3Mo are found in the spiral arms. They are more luminous than others. The average luminosity, we derived in present work, is based on the O-rich AGB stars located in the arms.

Georgelin, Y.M., Georgelin, Y.P., Sivan, J.P.,1976,A\&A,49,57.

Habing, H.J.,1988, A\&A,200,40.

van der Veen,W.E.C.J., Habing, H.J.,1988,A\&A, 194,125. 